\title{
Tim3 Is Upregulated and Protective in Nephrotoxic Serum Nephritis
}

Andrea Schroll, ${ }^{*}$ Kathrin Eller, ${ }^{\dagger}$ Julia M. Huber, ${ }^{\dagger}$ Igor M. Theurl, ${ }^{*}$ Anna M. Wolf, ${ }^{\ddagger}$ Günter Weiss, ${ }^{*}$ and Alexander R. Rosenkranz ${ }^{\dagger}$

From the Departments of Internal Medicine I- Clinical Immunology and Infectious Diseases," Internal Medicine IVNephrology and Hypertension, ${ }^{\dagger}$ and the Tyrolean Cancer Research Institute, Laboratory of Tumor Immunology, Innsbruck Medical University, Innsbruck, Austria

T cell immunoglobulin and mucin protein-3 (Tim3) is mainly expressed on the cell surface of $\mathrm{T}$-helper lymphocytes $\left(T_{H}\right)$ that negatively regulates $T_{H^{-}}$-type 1 $\left(\mathrm{T}_{\mathrm{H}^{-1}}\right)$ responses. Because blockade of Tim3 aggravates disease activity in $T_{H^{-1}}$-dependent diseases, we investigated whether Tim3 is involved in the pathogenesis of the $\mathbf{T}_{\mathbf{H}^{-1}}$-dependent nephrotoxic nephritis (NTS). We first evaluated Tim3 expression in mice after induction of nephrotoxic serum nephritis (NTS) and then studied the effects of anti-Tim 3 treatment toward the course of NTS for up to seven days. Whereas Tim3 expression was undetectable in control mice, we found significantly increased Tim3 expression in kidneys, but not in draining lymph nodes, at one, four, and eight weeks after induction of NTS. Tim3-expressing cells that infiltrated kidneys of mice subjected to NTS turned out to be $\mathrm{CD}^{+} \mathrm{T}$ cells rather than $\mathrm{CD8}^{+}$cytotoxic $\mathrm{T}$ cells and dendritic cells. Administration of a blocking anti-Tim3 antibody aggravated nephritis as shown by significantly increased albuminuria, respective histological changes, and increased expression of the kidney injury molecule lipocalin-2. In parallel, an increase of infiltrating $\mathbf{T}$ cells, macrophages, and macrophage pro-inflammatory cytokine formation as well as increased proliferation and apoptosis in kidneys of anti-Tim3treated mice was detected. Together, we provide the first evidence that Tim 3 is up-regulated in kidneys in NTS and that Tim3 exerts protective roles in the course of disease. (Am J Pathol 2010, 176:1716-1724; DOI: 10.2353/ajpath.2010.090859)

T-helper $\left(T_{H}\right)$ cells play an essential role for the pathogenesis of various autoimmune diseases. $\mathrm{T}_{\mathrm{H}^{-}}$- cells, which induce a pro-inflammatory immune response, have been associated with the pathogenesis of rheumatoid arthritis, type I diabetes, or Crohn disease, but more recently $T_{H^{-1}}$ cells have also gained interest as important effector cells in these experimental models. ${ }^{1,2}$ In contrast, $\mathrm{T}_{\mathrm{H}^{-}}$-2 cell activation is essential for the development of allergic asthma and host response toward parasitic infections. ${ }^{3,4}$ Whereas several mechanisms in the priming and differentiation of naïve $T$ cells have been elucidated, the pathways determining the functional activity of differentiated effector $\mathrm{T}$ cells are largely unknown

The $\mathrm{T}$ cell immunoglobulin and mucin domains (Tim) are a group of cell surface receptors differentially expressed on mature $T$ cells and macrophages that can control the functionality of $T$ cell subsets by inducing activating or apoptotic signals after interaction with specific ligands. ${ }^{5}$ Specifically, Tim3 is preferentially expressed on differentiated $\mathrm{T}_{\mathrm{H}^{-1}}$ cells,,${ }^{6,7}$ but also on cytotoxic $\mathrm{CD}^{+}{ }^{+} \mathrm{T}$ cells, $\mathrm{T}_{\mathrm{H}^{-}} 17$ cells, $\mathrm{CD} 4^{+} \mathrm{CD} 25^{+}$regulatory $\mathrm{T}$ cells (Tregs), dendritic cells, and mast cells. ${ }^{8-12}$ Thereby, Tim3 acts as a negative regulator of pro-inflammatory immune effector pathways. Accordingly, administration of a blocking anti-Tim3 antibody in a model of experimental allergic encephalomyelitis resulted in activation and expansion of macrophages in the brain and worsening of the disease. ${ }^{7}$ This observation could be traced back to direct cell-to-cell-interaction between differentiated $\mathrm{T}_{\mathrm{H}^{-1}}$ cells and $\mathrm{CD} 11 \mathrm{~b}^{+} / \mathrm{F} 4 / 80^{+}$macrophages, ${ }^{7}$ which was inhibited by Tim3. These findings were confirmed in a mouse model of experimental type I diabetes mellitus. ${ }^{11}$ In addition, these authors found that anti-Tim3 blockade dampened the antigen-specific immunosuppressive function of Tregs. ${ }^{11}$

Experimental nephrotoxic nephritis (NTS) is an established murine model to study glomerulonephritis. ${ }^{13}$ On

\footnotetext{
Supported by the Austrian research funds FWF (P-19964 to G.W.) and the Medizinischer Forschungsfond Innsbruck (to K.E.)

Accepted for publication December 14, 2009.

Address reprint requests to Dr. Günter Weiss, Internal Medicine I Clinical Immunology and Infectious Diseases, or Dr. Alexander R. Rosenkranz, Internal Medicine IV - Nephrology and Hypertension, Innsbruck Medical University, Anichstrasse 35, 6020 Innsbruck, Austria. E-mail: guenter.weiss@i-med.ac.at or alexander.rosenkranz@i-med.ac.at.
} 
the one hand, $\mathrm{CD}^{+}{ }^{+} \mathrm{T}$ effector cells mediate NTS thereby contributing to albuminuria and kidney damage. ${ }^{14} \mathrm{Ac}-$ cordingly, the depletion of the master switch gene of $\mathrm{T}_{\mathrm{H}^{-1}}$ cell differentiation, t-bet, attenuated the development of NTS in mice. ${ }^{15}$ On the other hand, Tregs, mast cells, and dendritic cells, all of which express Tim3, are also supposed to be centrally involved in the pathogenesis of NTS ${ }^{14,16,17}$ as $\mathrm{CD}^{+}{ }^{+} \mathrm{CD} 25^{+} \mathrm{FoxP}^{+}$Treg have been shown to be protective in this setting. ${ }^{14}$ Because Tregs and mast cells were mainly found in the regional draining lymph nodes, it was concluded that the regulation of the immune response in NTS takes place in secondary lymphoid organs rather than in the kidney. ${ }^{14,17}$ However, as Tim3 plays a central role in the functional control of immune cell populations, which are also involved in the pathogenesis of NTS, ${ }^{11}$ we were interested to evaluate the role of Tim3 in this experimental disease model. We thus studied the expression of Tim3 in this murine NTS model and examined whether modulation of Tim3 functionality would have an impact on the course of disease.

\section{Materials and Methods}

\section{Study Design}

Eight- to 12-week-old male C57BL/6 and BALBc mice obtained from Charles River (Sulzfeld, Germany) were used throughout the studies. Animals were maintained in a virus/antibody-free central animal facility of the Innsbruck Medical University. Nephrotoxic serum nephritis was induced as described previously. ${ }^{18}$ In brief, mice were pre-immunized subcutaneously with $2 \mathrm{mg} / \mathrm{ml}$ rabbit IgG (Jackson ImmunoResearch Laboratories Inc., West Grove, PA) dissolved in incomplete Freund adjuvant (Sigma, St. Louis, MI), and nonviable desiccated Mycobacterium tuberculosis H37a (Difco Laboratories, Detroit, MI). After 3 days, heat-inactivated rabbit anti-mouse glomerular basement membrane (GBM) antiserum was injected via the tail vein. BALBc mice received the twofold dose of antiserum as compared with C57BL/6 mice and thereby developed a comparable cytokine response in kidneys and lymph nodes (data not shown). BALBc mice received $150 \mu \mathrm{g}$ anti-TIM3 blocking antibody (eBiosciences, San Diego, CA $)^{19}$ or a rat IgG isotype control antibody intraperitoneally on the day of immunization. Three days later mice received an additional dose of $50 \mu \mathrm{g}$ anti-TIM3 blocking antibody or solvent intraperitoneally. Twenty-four hour urine samples were collected in metabolic cages at the indicated time points. All animal experiments were approved by Austrian veterinary authorities.

\section{Urinary Albumin, Urinary Creatinine, as well as Urinary and Serum Lipocalin-2 Detection}

Urinary albumin was determined by a double-sandwich ELISA (Abcam, Cambridge, MA) as reported previously. ${ }^{18}$ Urinary creatinine was quantified spectrophotometrically using a Picric acid-based method (Sigma, St. Louis, $\mathrm{MI})$. Urine and serum Lcn2 concentrations were performed using a commercially available ELISA kit obtained from R\&D (Quantikine HS ELISA Kit, Minneapolis, MN).

\section{Detection of Circulating Mouse Anti-Rabbit Immunoglobulins}

For detection of circulating mouse anti-rabbit IgG 96-well plates (Greiner, Kremsmuenster, Austria) were coated with $100 \mu \mathrm{g} / \mathrm{ml}$ rabbit IgG (Jackson ImmunoResearch Laboratories Inc.) in carbonate/bicarbonate buffer ( $\mathrm{pH}$ 9.5). After blockade blocked with 1\% BSA plates were incubated with serial-doubling dilutions of mouse serum. Bound mouse IgG was detected with HRP-conjugated goat-anti-mouse IgG (Dako, Glostrup, Denmark), IgG1, IgG2b, IgG3 (Jackson ImmunoResearch Laboratories Inc.) at a dilution of 1:25,600 for IgG and at a dilution of 1:1600 for IgG subclasses.

\section{Assessment of Glomerular Injury}

Formalin-fixed renal tissue was embedded in paraffin, cut in 4- $\mu \mathrm{m}$ sections, and stained with periodic acid Schiff (PAS) for histological analysis. In all cases, a minimum of 50 equatorial glomerular cross-sections were evaluated as described previously. ${ }^{20}$

For detection of anti-proliferating cell nuclear antigen (PCNA)-positive cells, we dehydrated paraffin sections and performed antigen retrieval by using a commercially available antigen-unmasking citric-acid buffer (Vector Laboratories, Burlingame, CA). For the staining procedure, we used the mouse anti-mouse PCNA antibody (BioLegend, San Diego, CA) as a primary antibody and the M.O.M. staining kit (Vector Laboratories) according to manufacturer's instructions.

The three-layer immunoperoxidase technique on frozen tissue sections ( $4 \mu \mathrm{m})$ was used for the detection of macrophages and T cells in the kidney sections. ${ }^{18}$ Macrophages were stained using two different rat anti-mouse antibodies, namely clone F4/80 (Serotec, Oxford, UK) and CD68 (Abcam) as described. ${ }^{21}$ A semiquantitative scoring system for F4/80-positive cells was used as follows: $0=0$ to 4 cells stained positive, $1+=5$ to 10 cells, $2+=10$ to 50 cells, $3+=50$ to 200 cells, and $4+=$ more than 200 cells stained positive per low-power field. For the detection of $\mathrm{CD}^{+}{ }^{+}$T-cells a rat anti-mouse CD4 (clone YTS191.1; Serotec) monoclonal antibody (mAb) was used. Biotin-conjugated goat anti-rat IgG antibody (Jackson ImmunoResearch Laboratories Inc., Cambridgeshire, UK) was used as a secondary antibody, followed by incubation with an avidin-biotin complex and subsequent development with $0.4 \%$ 3-amino-9-ethylcarbazole for 6 minutes and counterstaining with Gill Hematoxylin No. 3 (Polysciences Inc., Warrington, PA). Quantification of $\mathrm{T}$ cells and $\mathrm{CD}^{+} 8^{+}$cells was performed by counting the number of cells in six adjacent high-power fields of renal cortex and medulla. Additionally, intraglomerular infiltration of $\mathrm{F} 4 / 80^{+}$and $\mathrm{CD}^{+} 8^{+}$cells was performed by counting the number of positive cells in 25 glomeruli. 
Table 1. Primers and TaqMan Probes Used

\begin{tabular}{|c|c|c|c|}
\hline Gene & Forward primer & Reverse primer & Probe \\
\hline HPRT & $5^{\prime}-$ GACCGGTCCCGTCATGC-3' & $5^{\prime}$-TCATAACCTGGTTCATCATCGC-3' & 5'-ACCCGCAGTCCCAGCGTCGTC-3' \\
\hline TNF & $5^{\prime}-$-TTCTATGGCCCAGACCCTCA-3' & 5'-TTGCTACGACGTGGGCTACA-3' & 5'-CTCAGATCATCTTCTCAAAATTCGAGTGACAAGC-3' \\
\hline IL-6 & 5'-TGTTCTCTGGGAAATCGTGGA-3' & 5'-AAGTGCATCATCGTTGTTCATACA-3' & 5'-ATGAGAAAAGAGTTGTGCAATGGCAATTCTG-3' \\
\hline IL12p35 & $5^{\prime}$-TACTAGAGAGACTTCTTCCACAACAAGAG-3' & 5'-TCTGGTACATCTTCAAGTCCTCATAGA-3' & 5'-AGACGTCTTTGATGATGACCCTGTGCCT-3' \\
\hline IL12p40 & 5'-GACCATCACTGTCAAAGAGTTTCTAGAT-3' & 5'-AGGAAAGTCTTGTTTTTGAAATTTTTTAA-3' & 5'-CCACTCACATCTGCTGCTCCACAAGAAG-3' \\
\hline IFN- $\gamma$ & $5^{\prime}$-TCAAGTGGCATAGATGTGGAAGAA-3' & 5'-TGGCTCTGCAGGATTTTCATG-3' & 5'-TCACCATCCTTTTGCCAGTTCCTCCAG-3' \\
\hline t-bet & 5'-CCTGTTGTGGTCCAAGTTCAAC-3' & $5^{\prime}$-CACAAACATCCTGTAATGGCTTGT-3' & 5'-ATCATCACTAAGCAAGGACGGCGAATGTTCC-3' \\
\hline IL17A & 5'-GCTCCAGAAGGCCCTCAG-3' & 5'-CTTTCCCTCCGCATTGACA-3' & 5'-ACCTCAACCGTTCCACGTCACCCTG-3' \\
\hline$R O R \gamma t$ & $5^{\prime}-$ CCGCTGAGAGGGCTTCAC-3' & $5^{\prime}-$ TGCAGGAGTAGGCCACATTACA-3' & 5'-AAGGGCTTCTTCCGCCGCAGCCAGCAG-3' \\
\hline Foxp3 & $5^{\prime}$-AGGAGAAGCTGGGAGCTATGC-3' & $5^{\prime}-$ TGGCTACGATGCAGCAAGAG-3' & 5'-AGCGCCATCTTCCCAGCCAGG-3' \\
\hline GATA-3 & 5'-AGGAGAAGCTGGGAGCTATGC-3' & $5^{\prime}-$ TGGCTACGATGCAGCAAGAG-3' & $5^{\prime}-\mathrm{AGCGCCATCTTCCCAGCC}-3^{\prime}$ \\
\hline Tim-3 & 5'-GAACTGAAATTAGACATCAAAGCAGC- $3^{\prime}$ & 5'-GGTTCTTGGAGAAGCTGTAGTAGAGTC-3' & 5'-AGGTCACTCCAGCTCAGACTGCCCAT-3' \\
\hline Lon2 & $5^{\prime}$-GCCTCAAGGACGACAACATCA-3' & $5^{\prime}-$ CACCACCCATTCAGTTGTCAAT- $3^{\prime}$ & $5^{\prime}-\mathrm{TTCTCTGTCCCCACCGACCAATGC-3^{ \prime }}$ \\
\hline$\| 18$ & 5'-GACTCTTGCGTCAACTTCAAGGA-3' & 5'-TTGTCTGATTCCAGGTCTCCATT-3' & 5'-TGATGTTTATTGACAACACGCTTTACTTTATACCTGAAGA-3' \\
\hline
\end{tabular}

For the detection of heterologous IgG-deposition, sections were stained by direct immunofluorescence with a FITC-conjugated goat anti-rabbit IgG (Jackson ImmunoResearch Laboratories Inc.). Semiquantitative assessment of the glomerular deposition of IgG was performed by determining the end point positive titer for detection of staining using serial dilutions of each antibody. C3 deposition was evaluated by staining kidneys with a FITCconjugated goat anti-mouse C3 antibody (Cappel, ICN Pharmaceuticals, Aurora, Ohio).

\section{Quantitative RT-PCR}

Quantitative RT-PCR was performed as described. ${ }^{22}$ The cDNA was subjected to real-time PCR amplification using either TaqMan ${ }^{\circledR}$ Gene Expression Assays (Applied Biosystems, Foster City, CA) or Platinum SYBR ${ }^{\circledR}$ Green qPCR SuperMix-UDG (Invitrogen) together with the respective primer pairs: $\beta$ Actin forward: 5'-GAAGTGTGACGTTGACATCCG-3', reverse 5'-TGCTGATCCACATCTGCTGGA-3'; bcl-2 forward: 5'-GCATCTTCTCCTTCCAGCCT-3', reverse: 5'-CACCTACCCAGCCTCCGTTA-3'. TaqMan real-time PCR primers and probes were designed using the Primer Express Software from Applied Biosystems (Vienna, Austria). The TaqMan gene expression assay for CCR7 as purchased from Applied Biosystems. TaqMan probes were labeled with the reporter dye 6-carboxyflurescein (FAM) at the $5^{\prime}$-end and with black hole quencher 1 (BHQ1) at the $3^{\prime}-$ end. Primers and probes were purchased from Microsynth (Balgach, Switzerland). For quantification, $50 \mathrm{ng}$ of cDNA, $12.5 \mu \mathrm{l}$ of Brilliant QPCR Master-Mix (Stratagene, Amsterdam, The Netherlands), TaqMan probe, forward and reverse primer were combined in a $25-\mu$ l reaction. The PCR reaction was performed and analyzed on the MX4000 Quantitative PCR System (Stratagene). The primers and TaqMan probes used are listed in Table 1.

\section{Flow Cytometry Analysis}

Single cell suspensions of kidneys of mice subjected to NTS or healthy control mice were performed. Samples were stained for Tim3, CD3, CD4, CD8, and CD11c (all from BD, San Diego, CA) and analyzed on a BD FACSCalibur System (BD).

\section{Western Blotting}

Protein extracts were prepared from nitrogen frozen tissue, and Western blotting was performed as previously
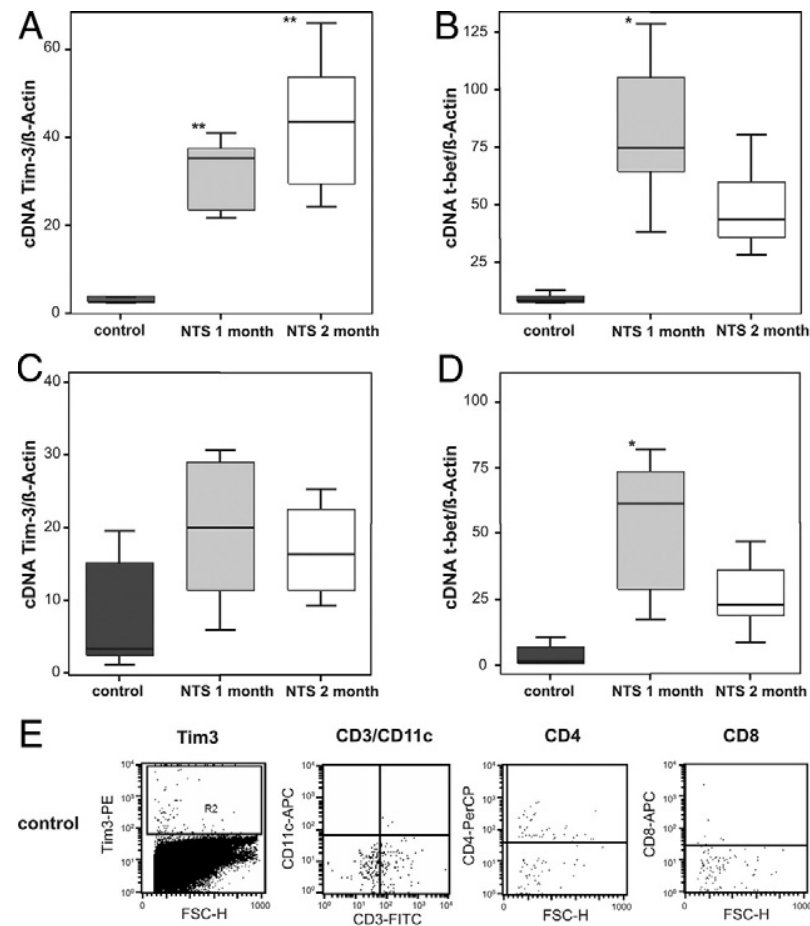

$\mathrm{CD} 3 / \mathrm{CD} 11 \mathrm{c}$
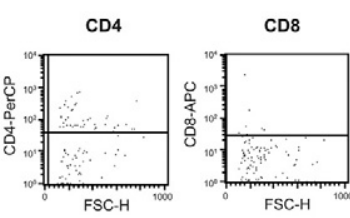

NTS
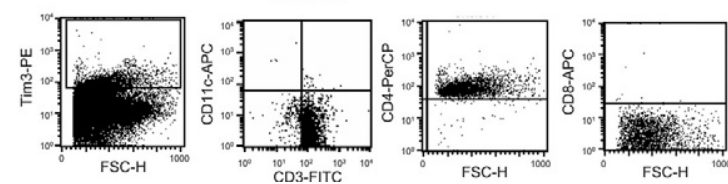

Figure 1. Tim 3 and t-bet expression are increased after induction of NTS in kidney. Total RNA was extracted from kidneys (A and $\mathbf{B})$ and the inguinal lymph node $(\mathbf{C}$ and $\mathbf{D})$ of $\mathrm{C} 57 \mathrm{BL} / 6$ mice for subsequent reverse transcription and quantitative real-time PCR at 4 and 8 weeks after induction of NTS. Cytoplasmatic mRNA levels of Tim3 (A and $\mathbf{C})$ and t-bet $(\mathbf{B}$ and $\mathbf{D})$ were determined by quantitative real-time PCR. Values were corrected for the amount of $\beta$-actin mRNA. Data are depicted as lower quartile, median, and upper quartile (boxes) and minimum/maximum ranges (whiskers). $n=4$ to 7 per group. ${ }^{*} P<0.05$ and *** $P<0.01$ compared with the control group as assessed by Mann-Whitney test. E: C57BL/6 mice were subjected to NTS and followed for 4 weeks. Single cell suspensions of kidneys of NTS mice and healthy controls were performed stained for Tim3, CD3, CD11c, CD4, and CD8, and analyzed by flow cytometry. Cells gated for Tim3 were analyzed for their expression of CD3, CD11c, CD4, and CD8. Representative dot plots from $n=3$ per group are shown. 
described, ${ }^{22}$ using PCNA ( $1 \mu \mathrm{g} / \mathrm{ml}$; BioLegend) or anti- $\beta$ Actin antibody (0.5 $\mu \mathrm{g} / \mathrm{ml}$; Sigma, Saint Louis, Missouri).

\section{In Situ Cell Death Detection}

Terminal transferase dUTP nick-end labeling (TUNEL) was performed using a commercially available staining kit (Roche, Basel, Switzerland) with the following changes to the recommended protocol. Glass slides were pretreated with Vectabond (Vector Laboratories) according to the manufacturer's protocol. After rehydratation tissue sections were treated with $10 \mu \mathrm{g} / \mathrm{ml}$ Proteinase K (Roche) and TUNEL mixture was applied onto each section. The slides were incubated in a humidified chamber for 60 minutes at $37^{\circ} \mathrm{C}$ in the dark. Finally, slides were rinsed three times in PBS. Positive controls were generated by incubating samples from control kidneys at room temperature for 10 minutes with $100 \mu \mathrm{l}$ of a $1000 \mathrm{U} / \mathrm{ml}$ solution of DNase I (Invitrogen) in DNase I buffer $(50 \mathrm{mmol} / \mathrm{L}$ Tris$\mathrm{HCl}, \mathrm{pH} 7.5,1 \mathrm{mg} / \mathrm{ml} \mathrm{BSA}$ ) to induce strand breaks. Sections were then washed three times in PBS. Negative controls were created by incubating sections with label solution alone. Nuclei were counterstained with 4',6-Diamidino-2-phenylindol, dihydochloride (DAPI; Molecular Probes Europe BV, Leiden, The Netherlands) at a concentration of $300 \mathrm{nmol} / \mathrm{L}$. TUNEL-positive cells per 20 high-power fields were quantified.

\section{Data Analysis}

Statistical analysis was performed using Statistics Package for the Social Science software package, version 15.1 (SAS Institute, Chicago, IL). Calculations for statistical differences between the various groups were performed by analysis of variance technique and Bonferroni correction for multiple tests, Student $t$ test, and MannWhitney technique.

\section{Results}

\section{Tim3 Expression Is Up-Regulated in Kidneys After Induction of NTS}

First, we evaluated Tim3 expression in kidneys and lymph nodes of C57BL/6 mice, which were subjected to NTS. We found a significantly increased expression of Tim3 mRNA in kidneys (Figure 1A) but only a moderate increase in lymph nodes (Figure 1C) at one and two month after induction of NTS. Additionally, we observed a significantly increase of t-bet expression in the kidney (Figure 1B) and in the draining lymph nodes (Figure 1D). Tim3 expression positively correlated with the mRNA expression of the master switch gene of $\mathrm{T}_{\mathrm{H}^{-1}}$ cells, t-bet, in the kidney (Spearman $r=0,65 ; P=0002$ ) as well as in the lymph nodes (Spearman $r=0,82, P<0001$ ). Flow cytometry analysis of kidneys revealed that the Tim3 infiltrating cells mainly expressed CD3 and were negative for CD11c. Further characterization revealed the $\mathrm{CD}^{+} \mathrm{Tim}^{+}$cells to express CD4 and not CD8 (Figure 1E).
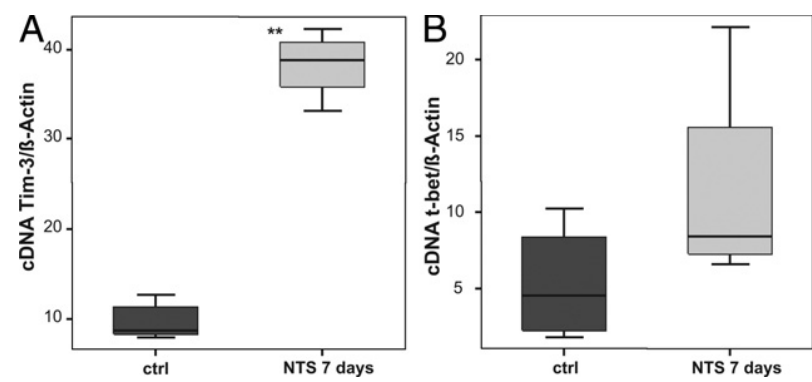

Figure 2. Tim 3 is already upregulated one week after NTS induction. Total RNA was extracted from kidney of $\mathrm{BALB} / \mathrm{c}$ mice for subsequent reverse transcription and quantitative real-time PCR one week after the induction of NTS. Cytoplasmatic mRNA levels of Tim3 (A) and t-bet (B) were determined by quantitative real-time PCR. Values were corrected for the amount of $\beta$-actin mRNA. Data are depicted as lower quartile, median, and upper quartile (boxes) and minimum/maximum ranges (whiskers). $n=5$ per group. ${ }^{*} P<0.05$ and ${ }^{* *} P<0.01$ compared with the control group as assessed by Mann-Whitney test.

\section{Tim3 Blockade Aggravates Renal Damage}

We then investigated the functional importance of Tim3 in vivo by using a blocking anti-Tim3 mAb. Because the commercially available blocking antibody is only efficient in BALBc mice, we first tested Tim3 regulation in BALBc mice subjected to NTS. Already one week after induction of disease we found significantly increased Tim3 expression in kidneys as compared with healthy controls (Figure $2 \mathrm{~A}$ ), which was paralleled by the t-bet expression (Figure 2B). No differences in Tim3 and t-bet expression were detected in lymph nodes (data not shown).

When the anti-Tim3 antibody was applied, we observed a significant increase in albuminuria seven days after induction of NTS as compared with mice injected with an isotype control antibody (healthy: $3 \pm 1 \mu \mathrm{g} / \mathrm{ml}$, NTS day 7: $664 \pm$ $181 \mu \mathrm{g} / \mathrm{mg}$, NTS + Tim3 mAb day 7: $2407 \pm 777 \mu \mathrm{g} / \mathrm{mg}$; Figure $3 A$ ). On histological examination, a mild to moderate hypercellularity and focal deposition of PAS-positive material (Figure 3C) were observed in mice receiving the isotype control, and these abnormalities were even enhanced in mice treated with the anti-Tim3 antibody (Figure 3D). Quantitation of PAS-positive areas revealed significant increased PAS-positive deposits in glomeruli of anti-Tim3 antibody treated and respective controls 7 days after induction of NTS (Figure 3B). Only marginal tubular changes were detected in both groups (data not shown).

Additionally, we evaluated the expression of lipocalin-2 (Lcn-2), a marker for kidney injury. ${ }^{13,23}$ Lcn2 mRNA was significantly increased in kidneys of anti-Tim3 antibody treated mice as compared with isotype treated controls at seven days after induction of the disease (Figure 4A). In accordance, we detected significantly increased Lcn2 levels in the urine (Figure 4B) and serum (Figure 4C) of anti-Tim3 antibody-treated mice.

\section{Effects of Tim3 Blockade on Local B and T Cell Responses in NTS}

Because the B cell response is of relevance in our model of NTS, ${ }^{24}$ we tested for putative differences of mouse-antirabbit IgG titers as a function of anti-Tim3 treatment. Seven 

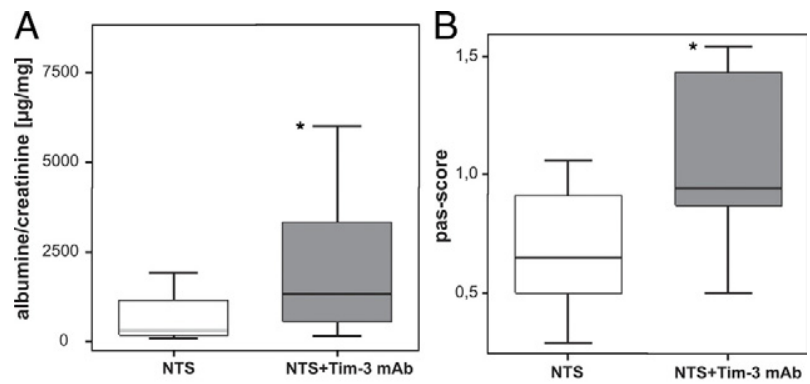

NTS

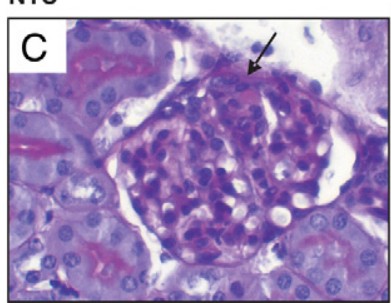

NTS+Tim-3 mAb

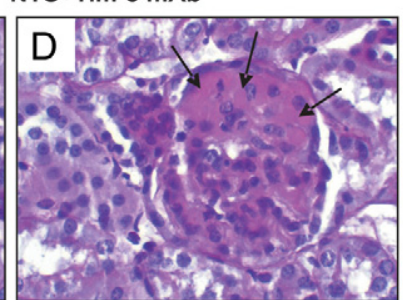

Figure 3. Blockade of Tim3 increases albumin secretion and aggravates morphological changes after induction of NTS. A: Treatment with anti-Tim3 antibody significantly increased albuminuria at day 7 after induction of NTS as compared with mice injected with the isotype control $(n=14) \cdot{ }^{*} P<0.05$ compared with control group determined by Mann-Whitney test. PASstained sections from day seven are shown. B: Quantification of PAS-positive deposits in mice receiving either Tim $3 \mathrm{mAb}$ or vehicle seven days after induction of NTS. ${ }^{*} P<0.05$ compared with control group determined by Mann-Whitney Test. A representative glomerulus from a mouse suffering from NTS for seven days showed a mild form from PAS-positive deposits $(\mathbf{C})$, whereas a representative glomerulus from a mouse that received Tim $3 \mathrm{mAb}$ showed enlargement and increased PAS-positive deposits (D). Magnification $\times 400$.

days after induction of NTS, no difference in the whole immunoglobulin and immunoglobulin subclasses lgG1 and IgG3 concentrations were found in mice treated with either a Tim3-blocking antibody or the respective isotype control (Table 2). Interestingly, Tim3-treated mice presented with significantly decreased mouse anti-rabbit IgG2b titers (Table 2). Of note, the deposition of the rabbit anti-mouse GBM antiserum (Table 2) and C3 deposition (data not shown) were comparable in both groups.

As this NTS model is a $T_{H^{-1}}$ dependent, in which $T_{\mathrm{H}^{-17}}$ cells might also be of importance, ${ }^{24}$ we next studied $\mathrm{T}$ cell function. Only very few $\mathrm{CD}^{+}{ }^{+} \mathrm{T}$ cells were found to
Table 2. Mouse Anti-Rabbit IgG Serum Titers and Deposition of Rabbit IgG on the GBM

\begin{tabular}{ccc}
\hline Parameter & NTS & NTS + Tim-3 mAb \\
\hline $\begin{array}{c}\text { Mouse anti-rabbit IgG } \\
\left(\mathrm{OD}_{450}\right)\end{array}$ & $0.82 \pm 0.08$ & $0.73 \pm 0.09$ \\
$\begin{array}{c}\text { Mouse anti-rabbit IgG1 } \\
\left(\mathrm{OD}_{450}\right)\end{array}$ & $0.99 \pm 0.15$ & $0.83 \pm 0.26$ \\
$\begin{array}{c}\text { Mouse anti-rabbit IgG2b } \\
\left(\mathrm{OD}_{450}\right)\end{array}$ & $0.86 \pm 0.10$ & $0.70 \pm 0.14^{*}$ \\
$\begin{array}{c}\text { Mouse anti-rabbit IgG3 } \\
\left(\mathrm{OD}_{450}\right)\end{array}$ & $0.51 \pm 0.07$ & $0.43 \pm 0.14$ \\
$\begin{array}{c}\text { Deposition of rabbit IgG } \\
\text { (titer) }\end{array}$ & $1: 3200$ & $1: 3200$ \\
\hline
\end{tabular}

Mice subjected to NTS received either an anti-Tim-3 antibody (NTS + Tim-3 mAb; $n=14$ ) or vehicle (GN; $n=14)$. After 7 days the mouse anti-rabbit IgG serum titers and immunoglobin subclass titers as well as the deposition of rabbit IgG on the GBM was evaluated. Data are shown as means $+\mathrm{SD}$.

${ }^{\star} P<0.05$

infiltrate kidneys 7 days after induction of NTS, but significantly higher numbers of $\mathrm{CD}^{+}{ }^{+} \mathrm{T}$ cells were found in anti-Tim3-treated mice as compared with isotype control-treated animals (Figure 5A). The few infiltrating $\mathrm{CD}^{+}{ }^{+} \mathrm{T}$ cells were mainly located intra- and periglomerularly (Figure 5, B and C). Seven days after induction of NTS real-time PCR revealed no difference in the expression of the Th-2 master switch gene GATA-3 as well as of the Th-1-specific genes T-bet and IFN- $\gamma$ in kidneys of anti-Tim3-treated mice and controls (Figure 5D). mRNA expression of interleukin (IL)-17A and ROR $\gamma$ T was increased in kidneys of antiTim3-treated mice compared with respective controls seven days after induction of NTS, but significance was not reached (Figure 5D).

Recently, we provided evidence that immune regulation in NTS mainly takes place in the regional draining lymph nodes rather than in the kidney. ${ }^{14}$ Interestingly, anti-Tim3 antibody administration did not have any effect on the $\mathrm{T}_{\mathrm{H}^{-1}}$ or $\mathrm{T}_{\mathrm{H}^{-}}-2$ cytokine and FoxP3 mRNA expression in lymph nodes (Table 3).

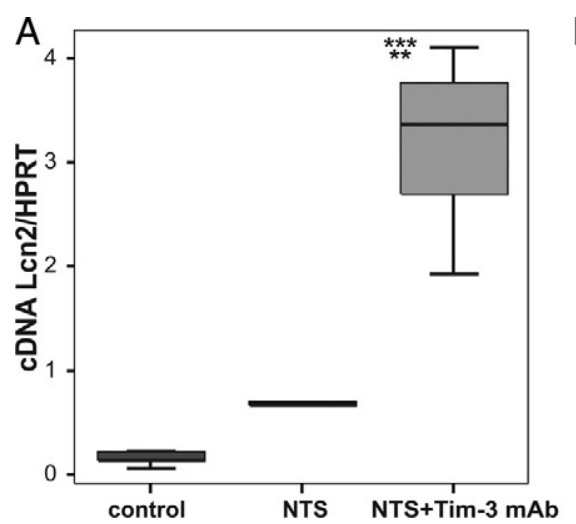

B

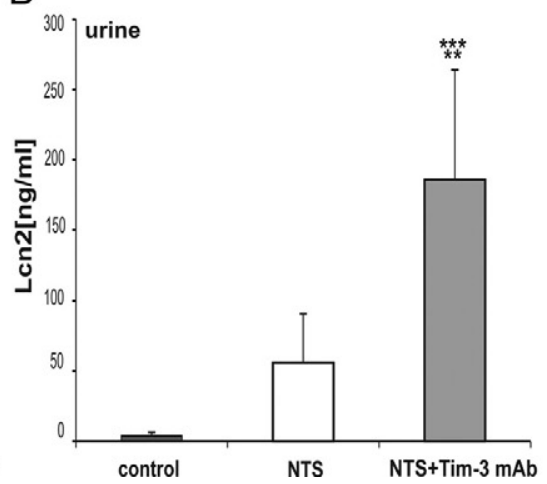

C

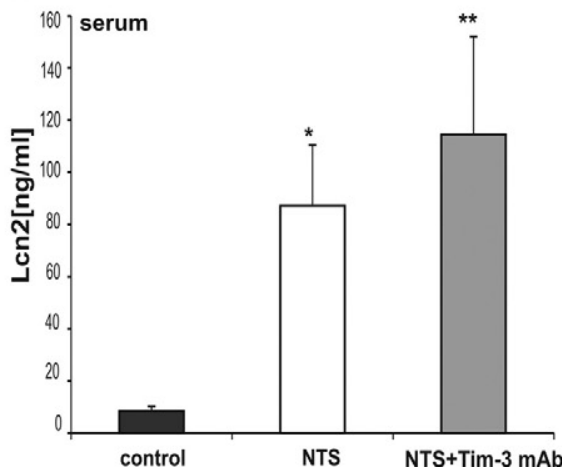

Figure 4. Tim3 blockade leads to a significant upregulation of Lcn2 expression. Total RNA was extracted from kidney of control, NTS, and NTS mice treated with an anti-Tim3 antibody. Quantification of Lcn2 mRNA levels (A) was performed by quantitative real-time PCR. Values were corrected for the amount of HPRT mRNA, measured in parallel. Data are depicted as lower quartile, median, and upper quartile (boxes) and minimum/maximum ranges (whiskers; A). Urine (B) and serum $(\mathbf{C})$ Lcn 2 concentrations were measured, and data are shown as means \pm SD of at least 5 mice; ${ }^{*} P<0.05$ and ${ }^{* *} P<0.01$ compared with the control group; ${ }^{* * * *} P<0.05$ compared with the NTS group, statistical calculation was performed by student $t$ test. 

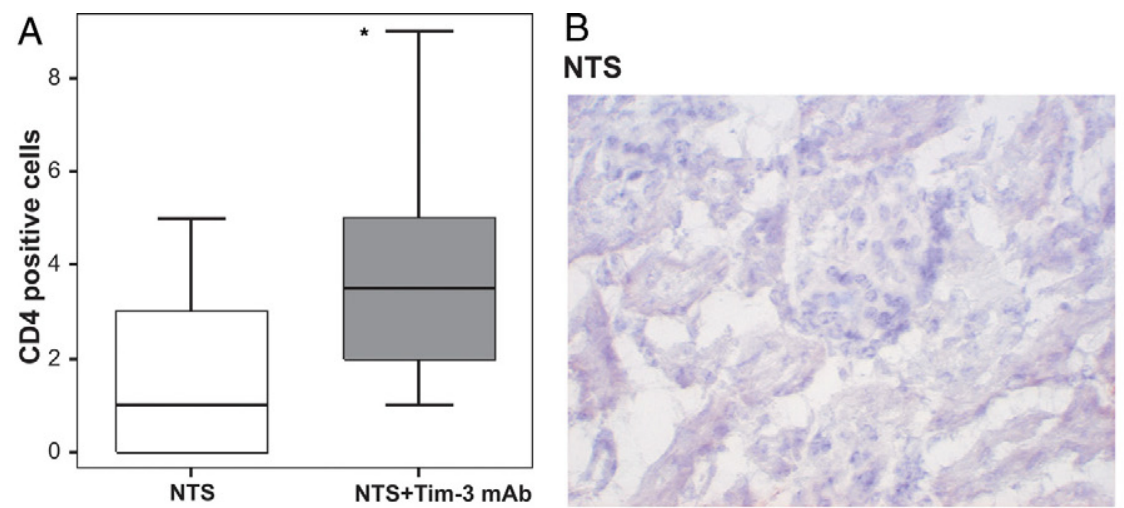

\section{C NTS+Tim-3 mAb}

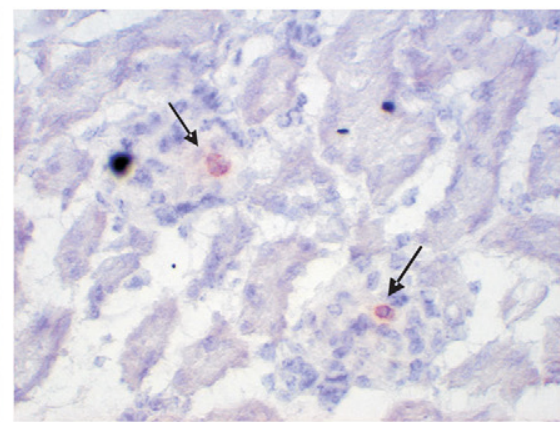

D

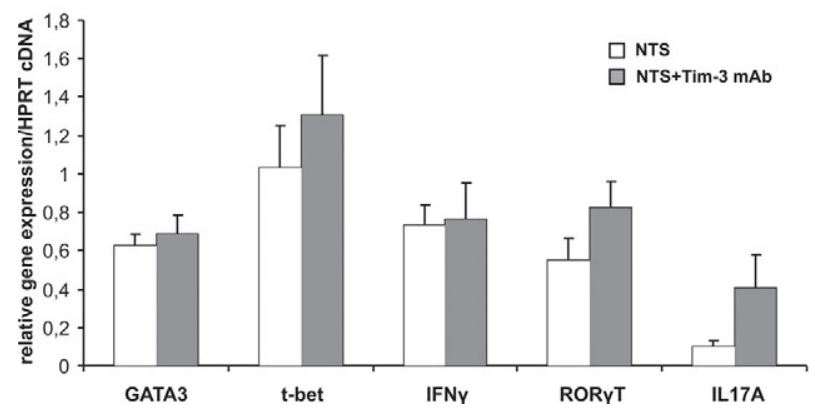

Macrophage Infiltration and Activity Is Significantly Increased in Kidneys After Tim3 Blockade

Macrophages are key effector cells in NTS. ${ }^{25}$ The number of cells positively stained for F4/80 (Figure 6A) and CD68 (Figure 6B) were significantly increased in the tubulointerstitium (Figure $6, \mathrm{~A}$ and $\mathrm{B}$ ) as well as intraglomerularly (Table 4) of kidneys of anti-Tim3 antibodytreated mice as compared with isotype-injected controls. $\mathrm{F} 4 / 80^{+}$cells were mainly found to infiltrate the interstitium and the periglomerular region, whereas $\mathrm{CD} 8^{+}$cells were mainly detected intraglomerularly (Figure 6, C-F). In line with the expansion of macrophage counts in the kidneys of anti-Tim3-treated mice, we observed an increase in markers of macrophage activation in these animals. Specifically, the expression of tumor necrosis

Table 3. Cytokine and FoxP3 mRNA Expression in Lymph Nodes

\begin{tabular}{lccc}
\hline Parameter & Control & NTS & NTS + Tim-3 mAb \\
\hline t-bet & $0.36 \pm 0.1$ & $1.40 \pm 0.5^{*}$ & $1.24 \pm 0.1^{*}$ \\
GATA-3 & $0.73 \pm 0.3$ & $0.68 \pm 0.2$ & $0.62 \pm 0.1$ \\
IL-10 & $0.35 \pm 0.2$ & $1.23 \pm 0.2^{*}$ & $1.02 \pm 0.4^{*}$ \\
IFN- $\gamma$ & $0.60 \pm 0.4$ & $4.8 \pm 0.6^{*}$ & $4.5 \pm 2.7^{*}$ \\
Foxp3 & $0.70 \pm 0.5$ & $1.05 \pm 0.5^{*}$ & $1.02 \pm 0.5^{*}$ \\
\hline
\end{tabular}

Mice received either an anti-Tim-3 blocking (NTS + Tim-3 mAb; $n=$ $14)$ or the respective vehicle (NTS; $n=14$ ) and were subjected to NTS. The control group did not receive any antibody and was not subjected to NTS $(n=4)$. After seven days lymph nodes were harvested and analyzed for the mRNA expression of $T_{H}-1$ and $T_{H}-2$ cytokines as well as for FoxP3. The relative cDNA expression of the respective genes after correction for the amount of the housekeeping gene HPRT mRNA is shown. Data are shown as means \pm SD.

*Significant differences as compared with the control, as determined by Student $t$ test. No significant differences were observed between the NTS and the NTS + TIM-3 mAb group.
Figure 5. Infiltration of $\mathrm{CD} 4^{+} \mathrm{T}$ cells and expression of effector pathways of Th-1 and Th-17 are affected by anti-Tim 3 antibody administration. The infiltration of the kidney by inflammatory cells was analyzed by immunohistochemistry. A: Kidneys were stained for $\mathrm{CD}^{+} \mathrm{T}$ cells seven days after induction of NTS in mice. Mean score of $\mathrm{CD}^{+}{ }^{+} \mathrm{T}$ cells as detailed in "Assessment of Glomerular Injury". Data are depicted as lower quartile, median, and upper quartile (boxes), and minimum/maximum ranges (whiskers). ${ }^{*} P<0.05$ when comparing the NTS with the NTS + Tim- 3 mAB group. Representative pictures of kidney sections of mice treated either with vehicle $(\mathbf{B})$ or anti-Tim $3 \mathrm{mAb}(\mathbf{C})$ seven days after induction of NTS stained for CD4 are shown. Magnification $\times 400$. Arrows mark CD4 ${ }^{+}$T cells. D: The mRNA expression of GATA-3, t-bet, IFN- $\gamma$, ROR $\gamma t$, and IL-17A were determined by quantitative real-time PCR. Values were corrected for the amount of Hprt mRNA, measured in parallel. Data are shown as means $\pm \mathrm{SD}$ of at least 5 mice.

factor $\alpha, \mathrm{IL}-6$, and IL-18 were significantly increased in kidneys of anti-Tim3 antibody-treated mice as compared with the respective controls at seven days after induction of NTS (Figure 6G). CCR7, which is known to be expressed in macrophages, $\mathrm{T}$ cells, and dendritic cells, ${ }^{26}$ as well as mesangial cells, ${ }^{27}$ was significantly increased in anti-Tim3 antibody-treated mice compared with controls (Figure 6G).

\section{Anti-Tim3 mAb Administration Leads to Increased Proliferation and Apoptosis}

To evaluate the cause of the severe kidney injury after Tim3 mAb administration, we hypothesized that Tim3 blockade on T cells may cause augmented proliferation of macrophages as shown in experimental allergic encephalomyelitis (EAE). ${ }^{7}$ PCNA protein was found to be significantly increased in kidney samples of mice treated with Tim3 mAb 7 days after induction of NTS (Figure 7, A and $\mathrm{B})$. By performing immunohistochemistry for the detection of PCNA, we found PCNA to be upregulated in interstitial and intraglomerular cell nuclei in anti-Tim3treated mice as compared with vehicle-treated controls, which might be assigned to an inflammatory cell population (Figure 7, C-E). In parallel, a significant downregulation of the anti-apoptotic bcl-2 mRNA (Figure 8A) and higher numbers of TUNEL-positive cells (Figure 8B) in anti-Tim3-treated mice as compared with respective controls were detected.

\section{Discussion}

Based on previous observations on the role of T-cells subsets, macrophages, and dendritic cells in the patho- 

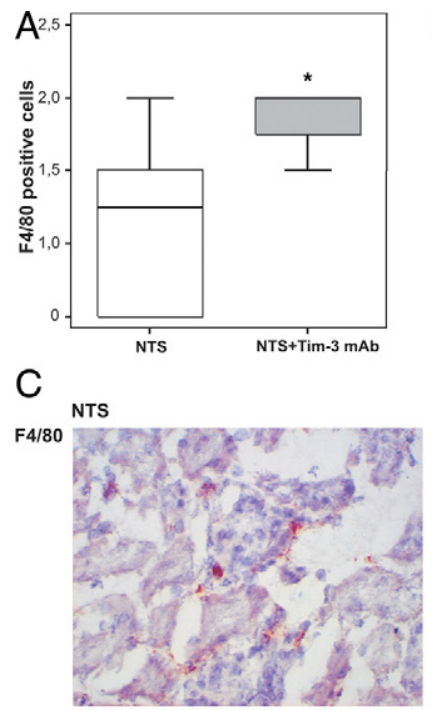

E

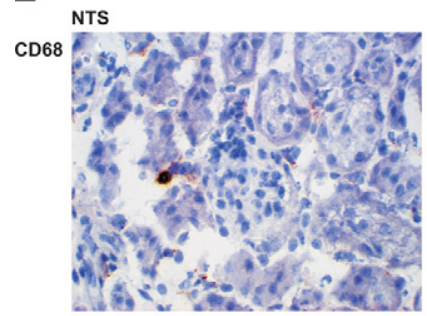

G

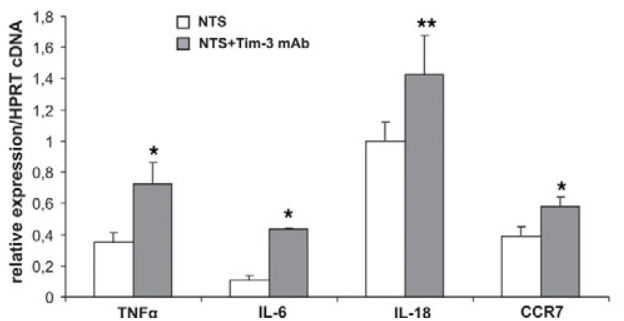

Figure 6. Macrophage infiltration and macrophage cytokine formation were significantly upregulated in anti-Tim 3 antibody-treated mice. The infiltration of macrophages and dendritic cells into kidneys was analyzed by immunohistochemistry for F4/80 (A) and CD68 (B) seven days after induction of NTS Representative pictures of kidney sections of mice treated either with (C and E) vehicle or (D and $\mathbf{F})$ anti-Tim $3 \mathrm{mAb}$ seven days after induction of NTS stained for $(\mathbf{C}$ and $\mathbf{D}) \mathrm{F} 4 / 80$ or $(\mathbf{E}$ and $\mathbf{F})$ CD68 are shown. Magnification $\times 400$. G: Quantitative TaqMan PCR for tumor necrosis factor, IL-6, and IL-18 RNA expression in the kidney was performed. Values are shown as relative cDNA abundance after normalization for Hprt cDNA expression. ${ }^{*} P<0.05$ and ${ }^{* *} P<0.001$ when comparing the NTS with the NTS + Tim 3 mAb group. Data are depicted as lower quartile, median, and upper quartile (boxes) and minimum/maximum ranges (whiskers; $\mathbf{A}$ and $\mathbf{B}$ ) or shown as means $\pm \mathrm{SD}$ (G) of at least five mice.

genesis of NTS ${ }^{8-12}$ and being aware of the fact that these immune cells potentially express Tim3, a cell surface receptor controlling T-cell and macrophage functionality, ${ }^{11}$ we herein explored the potential contribution of Tim3 to the immuno-pathogenesis of NTS. Tim3 was found to be significantly expressed in $\mathrm{CD}^{+}{ }^{+} \mathrm{T}$ cells infiltrating kidneys, but not in secondary lymphoid organs like the renal draining lymph nodes after induction of NTS. Blockade of Tim3 aggravated NTS by increasing macrophage infiltration into the kidneys as well as their activation status.
Table 4. Intraglomerular Infiltration of $\mathrm{F} 4 / 80^{+}$and $\mathrm{CD} 68^{+}$ Cells

\begin{tabular}{|c|c|c|}
\hline Parameter & NTS & $\mathrm{NTS}+\mathrm{Tim}-3 \mathrm{mAb}$ \\
\hline $\mathrm{F} 4 / 80^{+}$cells & $\begin{array}{r}6.80 \pm 2.04 \\
25.13+2.23\end{array}$ & $24.20 \pm 5.46^{*}$ \\
\hline
\end{tabular}

Mice received either an anti-Tim-3 blocking (NTS + Tim-3 mAb; $n=$ 14) or the respective vehicle (NTS; $n=14$ ) and were subjected to NTS. After seven days kidneys were harvested and stained for F4/80 and CD68. The number of positive cells in 25 glomerula is given. Data are shown as means $+\mathrm{SD}$.

*Significant differences, as determined by Student $t$ test.

Our data go along with the described role of Tim3 as a key suppressive signal on $\mathrm{T}_{\mathrm{H}^{-1}}$ cell-mediated immune function in experimental allergic encephalomyelitis (EAE) ${ }^{7}$ These authors found significant differences in macrophage infiltration into lesions in the central nervous system, whereas the $\mathrm{T}$ cell infiltration and cytokine production remained largely unaffected. ${ }^{7}$ In kidneys of mice subjected to NTS, the expression of Tim3 correlated with the mRNA expression of the master switch gene of $\mathrm{T}_{\mathrm{H}^{-}}$- cells $\mathrm{T}$-bet, but not with IL 17, suggesting that differentiated $\mathrm{T}_{\mathrm{H}^{-1}}$ rather than $\mathrm{T}_{\mathrm{H}^{-17}}$ cells are the main targets of Tim3 blockade.
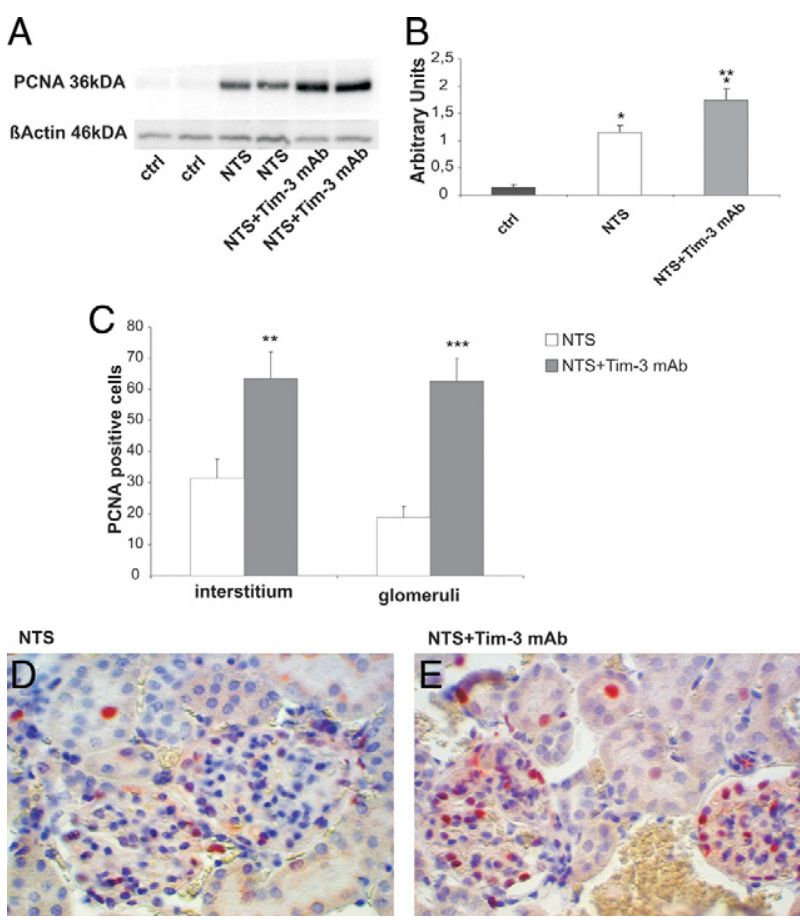

Figure 7. Tim $3 \mathrm{mAb}$ enhances proliferation in kidneys. Protein extracts from kidneys of control, NTS, and NTS + Tim3 mAb mice were analyzed for PCNA expression. A: A representative Western blot is shown. B: The expression of PCNA protein detected by Western blotting was quantified by using Quantity One Software $(n=4)$. ${ }^{*} P<0.05$ compared with control, ${ }^{* *} P<0.05$ compared with NTS; statistical calculation was performed by Mann-Whitney test. Additionally, PCNA was detected by immunohistochemistry. C: Positive cells were counted in the interstitium (number in six high-power fields) and in the glomeruli (number in 10 glomeruli). Data are shown as means $\pm \mathrm{SD}$ of at least 8 mice; ${ }^{* *} P<0.05$ and ${ }^{* * * * *} P<0.001$ compared with NTS. Calculations for statistical differences were performed by Mann-Whitney test. Representative PCNA stainings of kidney sections of mice treated either with vehicle (D) or anti$\operatorname{Tim} 3 \mathrm{mAb}(\mathbf{E})$ seven days after induction of NTS are shown. Magnification $\times 400$. 
A

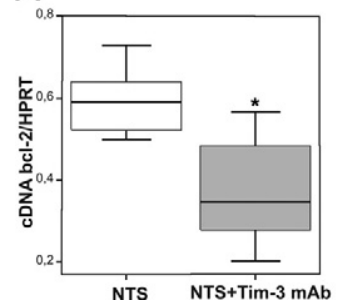

B

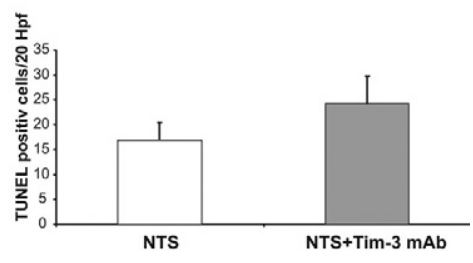

Figure 8. An increased number of immune cell activation reinforces apoptosis of kidney cells. A: Quantitative TaqMan PCR for detection of the anti-apoptotic gene bcl-2 was performed in kidneys of NTS and NTS + Tim 3 mAb mice 7 days after induction of NTS. Values are shown as relative cDNA abundance after normalization for HPRT cDNA expression. B: TUNEL assay was performed on paraffin-embedded sections, and apoptotic cells were quantified as TUNEL-positive cell per 20 high-power fields. ${ }^{*} P<0.05$ when comparing the NTS with the NTS+Tim 3 mAb group ( $n=$ at least 5 mice per group). Data are depicted as lower quartile, median, and upper quartile (boxes) and minimum/maximum ranges (whiskers; A) or shown as means \pm SD $(\mathbf{B})$ of at least 5 mice. Statistical calculation was performed by Mann-Whitney test.

Accordingly, Tim3 seems to act as a key inhibitory signal influencing either the recruitment or proliferation of macrophages, which are known effector cells in NTS. ${ }^{25}$ Nevertheless, it is largely unknown how Tim3 expressed on differentiated $\mathrm{T}_{\mathrm{H}^{-1}}$ cells or $\mathrm{T}_{\mathrm{H}^{-1}}$ cells regulates macrophage recruitment and activation. In experimental allergic encephalomyelitis (EAE) Tim3 blockade prevents apoptosis of macrophages and $\mathrm{T}_{\mathrm{H}^{-1}}$ cells after direct cell-to-cell-interaction between $\mathrm{T}_{\mathrm{H}^{-1}}$ cells and $\mathrm{CD} 11 \mathrm{~b}^{+} / \mathrm{F} 4 / 80^{+}$macrophages. ${ }^{7}$ However, it was also suggested that Tim3 may regulate macrophage function via modulation of Treg activity because Sanchez-Fueyo and coworkers have demonstrated that Tim3 blockade dampened the antigen-specific immunosuppressive function of Tregs in autoimmune diabetes mellitus type I. In NTS $\mathrm{CD} 4{ }^{+} \mathrm{CD} 25^{+}$Tregs have the capacity to inhibit NTS being located in the draining lymph nodes, whereas Tregs did not infiltrate the kidneys. ${ }^{14}$ After anti-Tim3 antibody treatment, we detected no significant differences in the infiltration of FoxP3 $^{+}$Tregs into draining lymph nodes. Nevertheless, we cannot rule out that the functionality of Tregs is impaired by Tim3 blockade.

A limitation of our study might be that we used, because of availability of the antibody, mice on BALB/C background. BALB/c mice are known to develop decreased disease indices and infiltration of $T$ cells and macrophages as compared with the usually used C57BL/6 mice. ${ }^{28}$ This is in accordance with our findings, but when the twofold dose of antiserum is used, BALB/C mice develop NTS with kidney infiltrates consisting of $T$ cells and macrophages comparable with C57BL/6 mice as has been shown recently. ${ }^{29}$

In NTS, two main compartments are involved in the pathogenesis of disease. On the one hand, immune initiation and regulation takes place in the draining lymph nodes, and on the other hand, the immune effector phase is located in the kidneys. Based on previous observations of our group, we suggested that mainly the immune regulation taking place in the secondary lymphoid organs $^{14,16,24}$ can protect mice from NTS, whereas immune regulations in the kidney may only affect the severity of the disease. Here, we only detected significantly increased Tim3 expression in kidneys of mice subjected to NTS, whereas no significant increase was found in the draining lymph nodes. In line, we found no influence of anti-Tim3 treatment on the expression of various cytokines in the regional draining lymph nodes. Thus, Tim3 blockade affects only the immune effector phase taking place locally in the kidney thereby modulating disease severity in NTS.

Lcn-2, a matrix peptide of human neutrophils that is also expressed in renal epithelial cells, has been described as a surrogate marker for acute and chronic kidney injury. ${ }^{23,30}$ Lcn-2 is significantly upregulated in nephritic animals and is further enhanced by anti-Tim3 treatment, both on mRNA as well as protein level. This also indicates that anti-Tim3 treatment does not only result in activation of macrophages in the kidneys but is also associated with aggravation of tissue damage. We could detect an increased PCNA proliferation signal in kidney tissue of Tim3-treated mice. Interestingly, significantly increased numbers of interstitial, tubular, as well as glomerular cells were PCNA-positive. The positive cells in the interstitium and in the glomeruli might be assigned to an inflammatory cell population. Because of the detected significant changes in macrophage infiltration as well as macrophage cytokine production we interpreted the increased proliferation to be mainly related to macrophages rather than to T cells or mesangial cells. Proliferating macrophages have been shown to be key effector cells in experimental NTS, ${ }^{31}$ but also in human glomerulonephritis. ${ }^{32}$ Nevertheless, further studies are necessary to characterize this population in more detail. The entity of immune cell proliferation, infiltration, and activation bred out a fatal damage of kidney function. We could only show a marginal increased number in TUNELpositive cells in anti-Tim3-treated mice, but a significant down-regulation in the mRNA expression of the anti-apoptotic factor bcl-2. Because we sacrificed mice seven days after induction of NTS, we would estimate that an extended period of antibody administration might probably show even more kidney cell apoptosis.

In summary, we provide evidence that Tim3 blockade aggravates NTS by increasing the number of kidney infiltrating inflammatory cells such as macrophages and promoting their activation as reflected by increased expression of macrophage-derived pro-inflammatory cytokines. Tim3 is expressed on differentiated effector $T_{H}$ cells in the kidney and thus negatively regulates the activation of macrophages in the kidney during NTS, and therefore stimulation of Tim3 expression may be a promising target for therapeutic intervention in autoimmune glomerulonephritis.

\section{Acknowledgments}

We are thankful for the brilliant technical assistance of Andrea Tagwerker and Lydia Markut. We are indebted to Afschin Soleiman for perfect advice in histological evaluations. 


\section{References}

1. Gutcher I, Becher B: APC-derived cytokines and T cell polarization in autoimmune inflammation. J Clin Invest 2007, 117:1119-1127

2. Mills $\mathrm{KH}$ : Induction, function and regulation of IL-17-producing $T$ cells. Eur J Immunol 2008, 38:2636-2649

3. Anderson GP, Coyle AJ: TH2 and 'TH2-like' cells in allergy and asthma: pharmacological perspectives. Trends Pharmacol Sci 1994, 15:324-332

4. Alexander J, Bryson K: T helper (h)1/Th2 and Leishmania: paradox rather than paradigm. Immunol Lett 2005, 99:17-23

5. Kuchroo VK, Umetsu DT, DeKruyff RH, Freeman GJ: The TIM gene family: emerging roles in immunity and disease. Nat Rev Immunol 2003, 3:454-462

6. Sabatos CA, Chakravarti S, Cha E, Schubart A, Sanchez-Fueyo A, Zheng XX, Coyle AJ, Strom TB, Freeman GJ, Kuchroo VK: Interaction of Tim-3 and Tim-3 ligand regulates T helper type 1 responses and induction of peripheral tolerance. Nat Immunol 2003, 4:1102-1110

7. Monney L, Sabatos CA, Gaglia JL, Ryu A, Waldner H, Chernova T, Manning S, Greenfield EA, Coyle AJ, Sobel RA, Freeman GJ, Kuchroo VK: Th1-specific cell surface protein Tim-3 regulates macrophage activation and severity of an autoimmune disease. Nature 2002, 415:536-541

8. Anderson AC, Anderson DE, Bregoli L, Hastings WD, Kassam N, Lei C, Chandwaskar R, Karman J, Su EW, Hirashima M, Bruce JN, Kane LP, Kuchroo VK, Hafler DA: Promotion of tissue inflammation by the immune receptor Tim-3 expressed on innate immune cells. Science 2007, 318:1141-1143

9. Nakae S, likura M, Suto H, Akiba H, Umetsu DT, Dekruyff RH, Saito H, Galli SJ: TIM-1 and TIM-3 enhancement of Th2 cytokine production by mast cells. Blood 2007, 110:2565-2568

10. Nakae S, Iwakura Y, Suto H, Galli SJ: Phenotypic differences between Th1 and Th17 cells and negative regulation of Th1 cell differentiation by IL-17. J Leukoc Biol 2007, 81:1258-1268

11. Sanchez-Fueyo A, Tian J, Picarella D, Domenig C, Zheng XX, Sabatos CA, Manlongat N, Bender O, Kamradt T, Kuchroo VK, Gutierrez-Ramos JC, Coyle AJ, Strom TB: Tim-3 inhibits T helper type 1-mediated autoand alloimmune responses and promotes immunological tolerance. Nat Immunol 2003, 4:1093-1101

12. Wang F, He W, Zhou H, Yuan J, Wu K, Xu L, Chen ZK: The Tim-3 ligand galectin-9 negatively regulates CD8+ alloreactive $T$ cell and prolongs survival of skin graft. Cell Immunol 2007, 250:68-74

13. Tipping PG, Holdsworth SR: T cells in glomerulonephritis. Springer Semin Immunopathol 2003, 24:377-393

14. Wolf $D$, Hochegger K, Wolf AM, Rumpold HF, Gastl G, Tilg H, Mayer G, Gunsilius E, Rosenkranz AR: CD4+CD25+ regulatory T cells inhibit experimental anti-glomerular basement membrane glomerulonephritis in mice. J Am Soc Nephrol 2005, 16:1360-1370

15. Phoon RK, Kitching AR, Odobasic D, Jones LK, Semple TJ, Holdsworth SR: T-bet deficiency attenuates renal injury in experimental crescentic glomerulonephritis. J Am Soc Nephrol 2008, 19:477-485

16. Hochegger K, Siebenhaar F, Vielhauer V, Heininger D, Mayadas TN, Mayer G, Maurer M, Rosenkranz AR: Role of mast cells in experimental anti-glomerular basement membrane glomerulonephritis. Eur J Immunol 2005, 35:3074-3082

17. Kruger T, Benke D, Eitner F, Lang A, Wirtz M, Hamilton-Williams EE, Engel D, Giese B, Muller-Newen G. Floege J, Kurts C: Identification and functional characterization of dendritic cells in the healthy murine kidney and in experimental glomerulonephritis. J Am Soc Nephrol 2004, 15:613-621

18. Rosenkranz AR, Mendrick DL, Cotran RS, Mayadas TN: P-selectin deficiency exacerbates experimental glomerulonephritis: a protective role for endothelial P-selectin in inflammation. J Clin Invest 1999, 103:649-659

19. Frisancho-Kiss S, Nyland JF, Davis SE, Barrett MA, Gatewood SJ, Njoku DB, Cihakova D, Silbergeld EK, Rose NR, Fairweather D: Cutting edge: $t$ cell Ig mucin-3 reduces inflammatory heart disease by increasing CTLA-4 during innate immunity. J Immunol 2006, 176: 6411-6415

20. Kitching AR, Holdsworth SR, Ploplis VA, Plow EF, Collen D, Carmeliet P, Tipping PG: Plasminogen and plasminogen activators protect against renal injury in crescentic glomerulonephritis. J Exp Med 1997, 185:963-968

21. Theurl M, Theurl I, Hochegger K, Obrist P, Subramaniam N, van Rooijen N, Schuemann K, Weiss G: Kupffer cells modulate iron homeostasis in mice via regulation of hepcidin expression. $\mathrm{J}$ Mol Med 2008, 86:825-835

22. Ludwiczek S, Theurl I, Muckenthaler MU, Jakab M, Mair SM, Theurl M, Kiss J, Paulmichl M, Hentze MW, Ritter M, Weiss G: Ca2 + channel blockers reverse iron overload by a new mechanism via divalent metal transporter-1. Nat Med 2007, 13:448-454

23. Bolignano D, Lacquaniti A, Coppolino G, Donato V, Campo S, Fazio MR, Nicocia G, Buemi M: Neutrophil gelatinase-associated lipocalin (NGAL) and progression of chronic kidney disease. Clin J Am Soc Nephrol 2009, 4:337-344

24. Hochegger K, Jansky GL, Soleiman A, Wolf AM, Tagwerker A, Seger C, Griesmacher A, Mayer G, Rosenkranz AR: Differential effects of rapamycin in anti-GBM glomerulonephritis. J Am Soc Nephrol 2008, 19:1520-1529

25. Duffield JS, Tipping PG, Kipari T, Cailhier JF, Clay S, Lang R, Bonventre JV, Hughes J: Conditional ablation of macrophages halts progression of crescentic glomerulonephritis. Am J Pathol 2005, 167:1207-1219

26. Forster R, Davalos-Misslitz AC, Rot A: CCR7 and its ligands: balancing immunity and tolerance. Nat Rev Immunol 2008, 8:362-371

27. Banas B, Wornle M, Berger T, Nelson PJ, Cohen CD, Kretzler M, Pfirstinger J, Mack M, Lipp M, Grone HJ, Schlondorff D: Roles of SLC/CCL21 and CCR7 in human kidney for mesangial proliferation, migration, apoptosis, and tissue homeostasis. J Immunol 2002, 168 4301-4307

28. Huang XR, Tipping PG, Shuo L, Holdsworth SR: Th1 responsiveness to nephritogenic antigens determines susceptibility to crescentic glomerulonephritis in mice. Kidney Int 1997, 51:94-103

29. Eller K, Weber T, Pruenster M, Wolf AM, Mayer G, Rosenkranz AR, Rot A: CCR7 deficiency exacerbates injury in acute nephritis due to aberrant localization of regulatory T cells. J Am Soc Nephrol 2010, 21:42-52

30. Mishra J, Dent C, Tarabishi R, Mitsnefes MM, Ma Q, Kelly C, Ruff SM Zahedi K, Shao M, Bean J, Mori K, Barasch J, Devarajan P: Neutrophil gelatinase-associated lipocalin (NGAL) as a biomarker for acute renal injury after cardiac surgery. Lancet 2005, 365:1231-1238

31. Lan HY, Nikolic-Paterson DJ, Mu W, Atkins RC: Local macrophage proliferation in the progression of glomerular and tubulointerstitial injury in rat anti-GBM glomerulonephritis. Kidney Int 1995, 48: 753-760

32. Yang N, Isbel NM, Nikolic-Paterson DJ, Li Y, Ye R, Atkins RC, Lan HY: Local macrophage proliferation in human glomerulonephritis. Kidney Int 1998, 54:143-151 